\title{
1 The core and the steady bargaining set for convex games
}

2 Josep Maria Izquierdo · Carles Rafels

4 Abstract Within the class of zero-monotonic and grand coalition superadditive

5 cooperative games with transferable utility, the convexity of a game is character-

6 ized by the coincidence of its core and the steady bargaining set. As a consequence

7 it is proved that convexity can also be characterized by the coincidence of the core

8 of a game and the modified Zhou bargaining set à la Shimomura.

9 Keywords cooperative game $\cdot$ convex games $\cdot$ core $\cdot$ bargaining set

\footnotetext{
The authors acknowledge the support from research grants ECO2014-52340-P (Ministerio de Economía y Competitividad) and 2014SGR40 (Generalitat de Catalunya).

J.M.Izquierdo

Facultat d'Economia i Empresa, Universitat de Barcelona, Av. Diagonal 690, Barcelona

Tel.: +34-934029090

Fax: $+34-934034892$

E-mail: jizquierdoa@ub.edu

C. Rafels
}

Facultat d'Economia i Empresa, Universitat de Barcelona, Av. Diagonal 690, Barcelona 


\section{0 \\ 1 Introduction}

11

12

18 the emptiness of the core, at a cost to be rather complex to compute. For this

Cooperative game theory analyzes how to distribute profits arising from the cooperation of a group of agents by proposing solutions that may consist on a unique allocation of those profits (payoff vector) or on a group of them meeting some stability conditions (set-solution). The core of a game $v, C(N, v)$, is the most natural set-solution concept but it might be empty. The bargaining sets (Davis and Maschler (1963, 1967), Mas-Colell (1989), Zhou (1994) and others) based on objections and counter-objections to payoff proposals offer an alternative solution to reason, it has been interesting to define non-empty subsolutions of the bargaining sets that were more simply to describe and check, that fulfill some stability conditions and that were related to the core of the game whenever it is non-empty. In this way, the first subsolutions we can find in the literature are the notion of quasi-core, introduced by Shapley and Shubik (1966), and the concept of kernel of a game (Davis and Maschler, 1965). Years after, Shimomura (1997) introduces the steady bargaining set of a game $v, \mathcal{S B}(N, v)$, and a small modification of the Shapley and Shubik quasi-core concept. The steady bargaining set of a game includes its core and it is a subsolution of two well-known variants of bargaining sets, also introduced by Shimomura (1997): the modified Mas-Colell bargaining set, $\mathcal{M B}^{*}(N, v)$, and the modified Zhou bargaining set, $\mathcal{Z}^{*}(N, v)$. The relationship among these solutions is as follows:

$$
C(N, v) \subseteq \mathcal{S B}(N, v) \subseteq \mathcal{Z}^{*}(N, v) \subseteq \mathcal{M B}^{*}(N, v)
$$

1 A sufficient condition that guarantees the non-emptiness of the steady bar32 gaining set and the modified Zhou bargaining set of a game is its grand coalition 

43 of a game by comparing its bargaining sets with the core, with special reference 44 to the stable bargaining set introduced by Greenberg (1992). Within the domain 45 of zero-monotonic games, Izquierdo and Rafels (2012) give a first answer to that 46 question by means of the coincidence of the core of a game and its modified Mas47 Colell bargaining set.

48 In this paper we focus on enriching the convexity characterization results. 49 Within the domain of zero-monotonic and grand coalition superadditive games, 50 the first characterization requires the coincidence of the the core of a game and its 51 steady bargaining set (Theorem 1). The elaborate proof of our new characterization 52 of the convexity of a game follows a two-step argument: first (see Proposition 1), 53 we show the characterization within a subclass of almost-convex games (introduced 54 by Núñez and Rafels, 1998); second, in Theorem 1, we tackle the general case. Finally, by the inclusion relationship given in (1), we also obtain as a corollary 56 of this theorem an additional new characterization of convex games in term of the 57 coincidence of the modified Zhou bargaining set and the core of the game (Corol- 
${ }_{58}$ lary 1). We expect this work might serve to both obtain new equivalence theorems

59 and to reanalyze the different convexity notions given for the non-transferable

60 utility case.

\section{Notations}

62 Let $N=\{1,2, \ldots, n\}$ be a set of players. For any coalition $S \subseteq N,|S|$ denotes

${ }_{63}$ the number of players in $S$. A cooperative game with player set $N$ is a function ${ }_{64} v: 2^{N} \rightarrow \mathbb{R}$ assigning to each coalition $S \subseteq N$ a real number $v(S)$ such that ${ }_{65} v(\varnothing)=0$. The function $v$ is called the characteristic function of the game and $v(S)$

66 is the worth of the coalition $S$. This number is interpreted as what the coalition ${ }_{67}$ can obtain on its own. Let $\mathcal{G}^{N}$ be the class of games with player set $N$. Given a 68 nonempty coalition $S \subseteq N$, we denote by $\left(S, v_{S}\right)$ the subgame of $(N, v)$ related to 69 $\quad$ coalition $S$ (i.e. $v_{S}(R)=v(R)$ for all $R \subseteq S$ ).

70 A game $v \in \mathcal{G}^{N}$ is monotonic if $v(S) \leq v(T)$, for any $S \subseteq T \subseteq N$. It is zero${ }_{71}$ monotonic if for all $S \subseteq T \subseteq N$ we have $v(S)+\sum_{i \in T \backslash S} v(\{i\}) \leq v(T)$ and it is grand 72 coalition zero-monotonic if for all $S \subseteq N$ we have $v(S)+\sum_{i \in N \backslash S} v(\{i\}) \leq v(N)$.

A game $v \in \mathcal{G}^{N}$ is superadditive if for all $S, T \subseteq N$ with $S \cap T=\varnothing$ it holds ${ }_{74} v(S)+v(T) \leq v(S \cup T)$, and it is grand coalition superadditive if for all partition $\mathcal{P}$ 75 of $N, \mathcal{P}=\left\{S_{1}, S_{2}, \ldots, S_{m}\right\}$, it holds that $\sum_{j=1}^{m} v\left(S_{j}\right) \leq v(N)$.

76 A game $v \in \mathcal{G}^{N}$ is convex if, for all $i \in N$,

$$
v(S \cup\{i\})-v(S) \leq v(T \cup\{i\})-v(T),
$$

77 for all $S \subseteq T \subseteq N \backslash\{i\}$. An equivalent definition of convexity states that, for all ${ }_{78} S, T \subseteq N$,

$$
v(S)+v(T) \leq v(S \cup T)+v(S \cap T) .
$$



corresponding subgame $\left(S, v_{S}\right)$ is convex.

Let $\mathbb{R}^{N}$ stand for the space of real-valued vectors $x=\left(x_{i}\right)_{i \in N}$ where $x_{i}$ is interpreted as the payoff to player $i \in N, x_{S}$ is the restriction of $x$ to the members

84 two vectors in $\mathbb{R}^{N}$, we write $x \geq y$ to mean that $x_{i} \geq y_{i}$, for all $i \in N$.

The set of preimputations of a game $v \in \mathcal{G}^{N}$ is defined by $I^{*}(N, v)=\{x \in$ $\left.{ }_{86} \mathbb{R}^{N} \mid x(N)=v(N)\right\}$. Its set of imputations is defined by $I(N, v)=\left\{x \in \mathbb{R}^{N} \mid x(N)=\right.$ ${ }_{87} v(N)$ and $x_{i} \geq v(\{i\})$, for all $\left.i \in N\right\}$ and its core is defined by $C(N, v)=\{x \in$ ${ }_{88} \quad \mathbb{R}^{N} \mid x(N)=v(N)$ and $x(S) \geq v(S)$ for all $\left.S \subseteq N\right\}$. A game with a non-empty 89 core is called a balanced game. Let $\mathcal{B}^{N} \subseteq \mathcal{G}^{N}$ be the subclass of balanced games 90 with player set $N$.

Given a game $v$, a preimputation $x \in I^{*}(N, v)$ and a pair of players $i$ and $j$, $i \neq j$, we define

$$
s_{i j}^{v}(x)=\max \{v(S)-x(S) \mid S \subseteq N, i \in S \text { but } j \notin S\} .
$$

We say that player $i$ outweigths player $j$ at $x$ if $s_{i j}^{v}(x)>s_{j i}^{v}(x)$. The prekernel of the game $v, \mathcal{P} \mathcal{K}(N, v)$, is the subset of preimputations such that no player outweights any other player at $x$. This is

$$
\mathcal{P K}(N, v)=\left\{x \in I^{*}(N, v) \mid \text { for all } i, j \in N, i \neq j, s_{i j}^{v}(x)=s_{j i}^{v}(x)\right\} .
$$

The kernel was introduced by Davis and Maschler (1965). It is based on the idea 93 of outweighting, but restricting the domain of feasible allocations to imputations. 94 A more general concept was analyzed by Schmeidler (1969) allowing to consider 
95 arbitrary payoff domains; in particular we are interested in those satisfying $Y=$ $Y(\ell, u)=\left\{x \in I^{*}(N, v) \mid \ell_{i} \leq x_{i} \leq u_{i}\right.$, for all $\left.i \in N\right\}$, where $\ell=\left(\ell_{i}\right)_{i \in N}$ and

${ }_{97} u=\left(u_{i}\right)_{i \in N}$, are the respective vectors of lower and upper bounds for the payoffs

Definition 1 The Mas-Colell bargaining set (à la Shimomura) is defined as

$$
\mathcal{M B}^{*}(N, v)=\left\{\begin{array}{l|l}
x \in I(N, v) & \begin{array}{l}
\text { for each objection to } x, \\
\text { there is a Mas-Colell's counterobjection }
\end{array}
\end{array}\right\} .
$$


Definition 2 The Zhou bargaining set (à la Shimomura) is defined as

$$
\mathcal{Z}^{*}(N, v)=\left\{\begin{array}{l|l}
x \in I(N, v) & \begin{array}{l}
\text { for each objection to } x \\
\text { there is a Zhou's counterobjection }
\end{array}
\end{array}\right\} .
$$

If no confusion arises we will refer to them simply as the Mas-Colell bargaining set and the Zhou bargaining set. By definition, these sets only consist on imputations (individually rational payoff vectors) and always includes the core. Shimomura (1997) states that a sufficient condition that guarantees the Mas-Colell bargaining set to be nonempty is grand coalition zero-monotonicity, while it is grand coalition superadditivity that ensures the non-emptiness of the Zhou bargaining set.

Shimomura also defines a subset of the Zhou bargaining set ( the steady bargaining set, $S B(N, v))$ by means of a dominant relationship between coalitions. He claims that the steady bargaining set can be rewritten as follows.

Definition 3 Let $v \in \mathcal{G}^{N}$ be a game. An imputation $x \in I(N, v)$ is in the steady bargaining set $\mathcal{S B}(N, v)$ if for all coalition $S \subseteq N$ with strictly positive excess $v(S)-x(S)>0$, there exists $M \subseteq N$, such that $S \backslash M \neq \varnothing, M \backslash S \neq \varnothing, S \cap M \neq \varnothing$ and $v(M)-x(M) \geq v(S)-x(S)$.

For any game $v$, it can be easily proved the inclusions $C(N, v) \subseteq \mathcal{S B}(N, v) \subseteq$ $\mathcal{Z}^{*}(N, v) \subseteq \mathcal{M B}^{*}(N, v)$; let us remark that these inclusions might be strict, even for superadditive games ${ }^{1}$. On the other hand, Izquierdo and Rafels (2012) show that

1 Let $N=\{1,2,3,4\}$ be the set of players and $v(N)=2, v(\{1,2,3\})=v(\{1,2,4\})=$ $v(\{1,3,4\})=v(\{2,3,4\})=1, v(\{1,3\})=v(\{1,4\})=v(\{2,3\})=1$ and $v(S)=0$, otherwise. Notice that the core of this game is non-emtpy. The payoff vector $x=\left(\frac{1}{2}, 1 \frac{1}{2}, 0,0\right) \in$ $\mathcal{M B}^{*}(N, v)$, but $x \notin \mathcal{Z}^{*}(N, v)$. Moreover, the payoff vector $x^{\prime}=\left(\frac{1}{2}, 1, \frac{1}{4}, \frac{1}{4}\right) \in \mathcal{Z}^{*}(N, v)$, but $x^{\prime} \notin C(N, v)$. With respect to the steady bargaining set notice $x^{\prime}=\left(\frac{1}{2}, 1, \frac{1}{4}, \frac{1}{4}\right) \in \mathcal{S} \mathcal{B}(N, v)$, but $x^{\prime} \notin C(N, v)$. The Example 2 (with $a=1$ ) in Shimomura (1997) provides an example of a 

do coincide, and thus, $C(N, v)=\mathcal{S B}(N, v)=\mathcal{Z}^{*}(N, v)=\mathcal{M B}^{*}(N, v)$.

Let $v \in \mathcal{B}^{N}$ be a balanced game and $\theta=\left(i_{1}, i_{2}, \ldots, i_{n}\right)$ be an ordering of players in $N$. We denote by $\Theta_{N}$ the set of all orderings in $N$. A marginal worth vector of the game $v$ relative to $\theta, m^{\theta}(v)$, is defined as

$$
\begin{aligned}
& m_{i_{1}}^{\theta}(v)=v\left(\left\{i_{1}\right\}\right) \text { and } \\
& m_{i_{k}}^{\theta}(v)=v\left(\left\{i_{1}, \ldots i_{k}\right\}\right)-v\left(\left\{i_{1}, \ldots i_{k-1}\right\}\right), \text { for all } k=2, \ldots, n .
\end{aligned}
$$

$$
v \text { is convex } \Leftrightarrow m^{\theta}(v) \in C(N, v) \text {, for all } \theta \in \Theta_{N} \text {. }
$$

We say that vector $x \in \mathbb{R}^{N}$ lexicographically precedes vector $y \in \mathbb{R}^{N}$ with respect to $\theta=\left(i_{1}, i_{2}, \ldots, i_{n}\right) \in \Theta_{N}, x \prec_{\ell}^{\theta} y$, if there exists $k \in\{1,2, \ldots, n\}$ such that $x_{i_{r}}=y_{i_{r}}$ for all $r=1, \ldots, k-1$ and $x_{i_{k}}<y_{i_{k}}$. The lexmin solution over the core of a balanced game $v \in \mathcal{B}^{N}$ relative to $\theta \in \Theta_{N}$ is defined as the (unique) payoff vector $\ell^{\theta}(v) \in C(N, v)$ that lexicographically precedes w.r.t. to $\theta$ any other vector in the core of the game $v$, i.e. $\ell^{\theta}(v) \prec_{\ell}^{\theta} x$ for all $x \in C(N, v)$. Let us remark that if a game $v$ is convex then $\ell^{\theta}(v)=m^{\theta}(v)$, for each ordering $\theta \in \Theta_{N}$.

\section{Characterization results}

In this section we provide two new characterizations of the convexity of a game.

The first one compares the steady bargaining set of the game with its core. To this aim, we first analyze the particular case of almost convex games (games superadditive game where the steady bargaining set is strictly included in the Zhou bargaining set. 

there exists a pair of players, say player 1 and player 2 such that

$$
v(N)-v(N \backslash\{1\})<v(N \backslash\{2\})-v(N \backslash\{1,2\}) .
$$

164 From Núñez and Rafels (1998) we know there is an extreme point $x \in C(N, v)$ of where all proper subgames are convex), since the argument used in the proof of the general case does not apply.

The second characterization is a direct consequence of the first one, and focuses on the coincidence of the Zhou bargaining set with the core.

Proposition 1 Let $(N, v)$ be a grand coalition superadditive and almost convex game. Then, the following statements are equivalent:

1. $v$ is convex.

2. $\mathcal{S B}(N, v)=C(N, v)$.

Proof 1. $\rightarrow$ 2.) From Izquierdo and Rafels (2012) it follows that, for any convex game $v, C(N, v)=\mathcal{M B}^{*}(N, v)$. Hence, since $C(N, v) \subseteq \mathcal{S B}(N, v) \subseteq \mathcal{M B}^{*}(v)$, we conclude $C(N, v)=\mathcal{S B}(N, v)$.

2. $\rightarrow$ 1.) As the game $v$ is grand coalition superadditive, the steady bargaining set is nonempty, i.e. $\mathcal{S B}(N, v) \neq \varnothing$ (Shimomura, 1997), and thus (by hypothesis), $\mathcal{S B}(N, v)=C(N, v) \neq \varnothing$. Therefore, the game is balanced. At this point, the proof is done for the two-person case, $n=2$, since any two-person balanced game is convex. Hence, from now on let us assume $n \geq 3$. Suppose to the contrary that the game is not convex. Since the game is almost convex but not convex, this means the core of the game $v$ such that ${ }^{2}$

$$
x_{1}=v(N)-v(N \backslash\{1\}) \text { and } x_{2}=v(N)-v(N \backslash\{2\}) .
$$

\footnotetext{
2 These authors prove that particular payoff vectors constructed based upon orderings of players $\theta=\left(i_{1}, i_{2}, \ldots, i_{n}\right)$ (the reduced marginal worth vector $\mathrm{rm}^{\theta}(v)$ ) are the extreme core
} 
By (5) and (6) it holds that

$$
\begin{aligned}
x_{1} & =v(N)-v(N \backslash\{1\})<v(N \backslash\{2\})-v(N \backslash\{1,2\}) \\
& =v(N)-(v(N)-v(N \backslash\{2\}))-v(N \backslash\{1,2\}) \\
& =v(N)-x_{2}-v(N \backslash\{1,2\})=x(N \backslash\{2\})-v(N \backslash\{1,2\}) .
\end{aligned}
$$

We conclude, $x(N \backslash\{1,2\})>v(N \backslash\{1,2\})$. Hence, let us remark now that the vector $x$ restricted to $N \backslash\{1,2\}, x_{N \backslash\{1,2\}}$, can be viewed as an aspiration of the subgame $^{3}\left(N \backslash\{1,2\}, v_{N \backslash\{1,2\}}\right)$. Since this subgame is convex and any convex game has a large core ${ }^{4}$ (Sharkey 1982), there exists $z \in C\left(N \backslash\{1,2\}, v_{N \backslash\{1,2\}}\right)$ such that $x_{N \backslash\{1,2\}} \geq z$. Moreover, since $x(N \backslash\{1,2\})>v(N \backslash\{1,2\})=z(N \backslash\{1,2\})$ there exists a player in $N \backslash\{1,2\}$, say player 3 such that $x_{3}>z_{3}$. This implies that

$$
x(S)>z(S) \geq v(S), \text { for all } S \subseteq N \backslash\{1,2\} \text { and } 3 \in S .
$$

Next define the vector $x^{\prime} \in \mathbb{R}^{N}$ as follows:

$$
x_{1}^{\prime}=x_{1}+\frac{\varepsilon}{2} ; x_{2}^{\prime}=x_{2}+\frac{\varepsilon}{2} ; x_{3}^{\prime}=x_{3}-\varepsilon \text { and } x_{k}^{\prime}=x_{k} \text { for all } k \in N \backslash\{1,2,3\},
$$

172 where $0<\varepsilon<\min _{3 \in S \subseteq N \backslash\{1,2\}}\{x(S)-v(S)\}$.

By the definition of $\varepsilon$ the vector $x^{\prime}$ is an imputation of the game $v$, but it is not a core element of $(N, v)$ since $x_{1}^{\prime}>x_{1}=v(N)-v(N \backslash\{1\})$ and so $x^{\prime}(N \backslash\{1\})<$ $v(N \backslash\{1\})$. However, for any coalition $S \subseteq N$ such that $v(S)-x^{\prime}(S)>0$ it is easy to check that:

elements of an almost convex balanced game. In particular, if $\theta=(1,2, \ldots, n)$, we have $r m_{1}^{\theta}(v)=v(N)-v(N \backslash\{1\})$ and $r m_{2}^{\theta}(v)=\min \{v(N \backslash\{1\})-v(N \backslash\{1,2\}), v(N)-v(N \backslash\{2\})\}=$ $v(N)-v(N \backslash\{2\})$, where the last equality follows from (5).

${ }^{3}$ An aspiration of a game $(N, v)$ is a vector $x^{\prime} \in \mathbb{R}^{N}$ satisfying all cores inequalities, i.e. $x^{\prime}(S) \geq v(S)$, for all $S \subseteq N$.

${ }^{4}$ A game has a large core if any aspiration $x^{\prime}$ of the game can be represented by a core allocation $x$, i.e. there exists $x \in C(N, v): x_{i} \leq x_{i}^{\prime}$, for all $i \in N$. 
177

178

179

(a) player $3 \in S$;

(b) either player $1 \in S$ or $2 \in S$, but not both;

(c) $v(S)-x^{\prime}(S) \leq \frac{\varepsilon}{2}$; and

(d) $v(N \backslash\{1\})-x^{\prime}(N \backslash\{1\})=v(N \backslash\{2\})-x^{\prime}(N \backslash\{2\})=\frac{\varepsilon}{2}$.

Taking these remarks into account, let $S \subseteq N$ be an arbitrary coalition with positive excess at $x^{\prime}$, i.e. $v(S)-x^{\prime}(S)>0$. We next show that there exists a coalition $M \subseteq N$ such that $M \cap S \neq \varnothing, S \backslash M \neq \varnothing, M \backslash S \neq \varnothing$ and $v(M)-x^{\prime}(M) \geq v(S)-x^{\prime}(S)$, and so that $x^{\prime}$ is in the steady bargaining set of $(N, v)$. We consider two cases:

A: If player $1 \in S$, take $M=N \backslash\{1\}$. Notice that, by (c) and (d), $v(M)-x^{\prime}(M)=$ $\frac{\varepsilon}{2} \geq v(S)-x^{\prime}(S)$. Moreover, by (a), player $3 \in M \cap S$, player $1 \in S \backslash M$ and, by (b), player $2 \in M \backslash S$.

B: If player $2 \in S$, take $M=N \backslash\{2\}$ and using an analogous reasoning we get that $v(M)-x^{\prime}(M)=\frac{\varepsilon}{2} \geq v(S)-x^{\prime}(S)$ with player $3 \in M \cap S$, player $2 \in S \backslash M$ and player $1 \in M \backslash S$.

We conclude the allocation $x^{\prime}$ is not a core element of the game $v$ but belongs to its steady bargaining set, i.e. $x \in \mathcal{S B}(N, v)$, which contradicts our hypothesis. Hence, the game $v$ must be convex.

The above characterization result can be now extended to a larger class of cooperative games. The thread of the proof of this result relies on the fact that for a convex game all marginal worth vectors are core elements and coincide with the corresponding lexmin solution relative to the different orderings. As a consequence, if a game is not convex there is at least one marginal worth vector that differs from the corresponding lexmin solution; based upon this, we will construct a particular vector not in the core but in the steady bargaining set. That is, we shall prove that 
(i) $\ell_{j_{k}}^{\theta}(v)=m_{j_{k}}^{\theta}(v)$, for all $\theta=\left(j_{1}, j_{2}, \ldots, j_{n}\right) \in \Theta_{N}$ and $k=1, \ldots, t^{*}-1$;

(ii) there exists $\theta^{*}=\left(i_{1}, i_{2}, \ldots, i_{n}\right) \in \Theta_{N}$ such that $\ell_{i_{t^{*}}}^{\theta^{*}}(v) \neq m_{i_{t^{*}}}^{\theta^{*}}(v)$.

216 Item $(i)$ indicates that, for all ordering, the payoff of players occupying the first

if a game is not convex the steady bargaining set of the game strictly includes its core. The proof of this result is constructive in the sense that, for any non-convex game satisfying conditions of Theorem 1, we built an allocation that turns out to be in the steady bargaining set of the game, but not in its core.

Theorem 1 Let $(N, v)$ be a zero-monotonic and grand coalition superadditive game. Then, the following statements are equivalent:

1. $v$ is convex.

2. $\mathcal{S B}(N, v)=C(N, v)$.

Proof 1. 2.) By convexity of the game $v$, it holds $C(N, v)=\mathcal{M B}^{*}(N, v)$ and thus, by (1), we conclude $C(N, v)=\mathcal{S B}(N, v)$.

$2 . \rightarrow 1$.) As the game $v$ is grand coalition superadditive, the steady bargaining set is nonempty, i.e. $\mathcal{S B}(N, v) \neq \varnothing$ (Shimomura, 1997), and thus (by hypothesis), $\mathcal{S B}(N, v)=C(N, v) \neq \varnothing$. Therefore, the game is balanced and the lexmin solution $\ell^{\theta}(v)$ is well-defined for all $\theta \in \Theta_{N}$. Let us suppose now that the game is not convex. Then, by (4), there must exist at least one ordering $\theta=\left(i_{1}, i_{2}, \ldots, i_{n}\right) \in \Theta_{N}$ such that $\ell^{\theta}(v) \neq m^{\theta}(v)$. Now, if we pairwise compare the lexmin vector $\ell^{\theta}(v)$ and the marginal worth vector $m^{\theta}(v)$ corresponding to all orderings we can determine a unique index $t^{*} \in\{1, \ldots, n\}$ satisfying that: $t^{*}-1$ positions coincide for both the lexmin vector and the marginal worth vector (notice that this condition does not impose any restriction when $t^{*}=1$ ); item 
(ii) states the existence of an ordering where the corresponding lexmin vector and marginal worth vector differ for the first time at position $t^{*}$.

Notice that, by Proposition 1, we may assume that $(N, v)$ is not an almost convex game and thus $t^{*} \neq n$. Moreover, as $\ell^{\theta^{*}}(v)$ is a core element, it can be checked in condition (ii) that

$$
\ell_{i_{t^{*}}}^{\theta^{*}}(v)>m_{i_{t^{*}}}^{\theta^{*}}(v)
$$

To prove it, in other case and by $(8), \ell_{i_{t^{*}}}^{\theta^{*}}(v)<m_{i_{t^{*}}}^{\theta^{*}}(v)=v\left(\left\{i_{1}, \ldots, i_{t^{*}}\right\}\right)-$ $v\left(\left\{i_{1}, \ldots, i_{t^{*}-1}\right\}\right)=v\left(\left\{i_{1}, \ldots, i_{t^{*}}\right\}\right)-m^{\theta^{*}}(v)\left(\left\{i_{1}, \ldots, i_{t^{*}-1}\right\}\right)=v\left(\left\{i_{1}, \ldots, i_{t^{*}}\right\}\right)-$ $\ell^{\theta^{*}}(v)\left(\left\{i_{1}, \ldots, i_{t^{*}-1}\right\}\right)$, which involves a contradiction since $\ell^{\theta^{*}}(v) \in C(N, v)$. Finally, we can also deduce from the above condition $(i)$ that,

for all $S \subseteq N$ with $|S|<t^{*}$, the subgame $\left(S, v_{S}\right)$ is convex.

Now, define

$$
\mathcal{S}^{\theta^{*}}(v)=\left\{M \subseteq N \mid M \neq N, i_{t^{*}} \in M \text { and } \ell^{\theta^{*}}(v)(M)=v(M)\right\},
$$

where $\theta^{*}=\left(i_{1}, i_{2}, \ldots, i_{n}\right)$ is given in $(i i)$ of $(8)$.

We claim $\mathcal{S}^{\theta^{*}}(v) \neq \varnothing$. Otherwise, $\ell^{\theta^{*}}(v)(M)>v(M)$, for all $M \subseteq N, M \neq N$, and $i_{t^{*}} \in M$. Taking this into account we might define the allocation $x \in \mathbb{R}^{N}$ as $x_{i_{t^{*}}}=\ell_{i_{t^{*}}}^{\theta^{*}}(v)-\varepsilon_{1}, x_{i_{t^{*}+1}}=\ell_{i_{t^{*}+1}}^{\theta^{*}}(v)+\varepsilon_{1}$ and $x_{i_{k}}=\ell_{i_{k}}^{\theta^{*}}(v)$, else, where $0<\varepsilon_{1}<$ $\min _{M \nsubseteq N, i_{t^{*}} \in M}\left\{\ell^{\theta^{*}}(v)(M)-v(M)\right\}$, and prove that $x \in C(N, v)$. However, this would contradict $\ell^{\theta^{*}}(v)$ to be the lexmin solution relative to $\theta^{*}$ over the core of the game $v$.

Let us denote by $\mathcal{S}_{\min }^{\theta^{*}}(v)$ the set of minimal coalitions with respect to the inclusion in the ordered set $\left(\mathcal{S}^{\theta^{*}}(v), \subseteq\right)$ and by $T^{*}$ the first $t^{*}$ agents of the ordering 
$\theta^{*}=\left(i_{1}, \ldots, i_{n}\right)$ given in $(8)$, i.e. $T^{*}=\left\{i_{1}, \ldots, i_{t^{*}}\right\}$. Notice that

$$
\ell^{\theta^{*}}(v)\left(T^{*}\right)>v\left(T^{*}\right)
$$

238 where the strict inequality follows from (8) and (9), since $\ell^{\theta^{*}}(v)\left(T^{*}\right)=\ell^{\theta^{*}}(v)\left(T^{*} \backslash\right.$

$\left.239\left\{i_{t^{*}}\right\}\right)+\ell_{i_{t^{*}}}^{\theta^{*}}(v)=m^{\theta^{*}}(v)\left(T^{*} \backslash\left\{i_{t^{*}}\right\}\right)+\ell_{i_{t^{*}}}^{\theta^{*}}(v)>m^{\theta^{*}}(v)\left(T^{*}\right)=v\left(T^{*}\right)$.

240 Next, it can be shown that the set $\mathcal{S}_{\min }^{\theta^{*}}(v)$ contains at least two coalitions.

241 This result is stated in Claim 1 but the rather technical proof is consigned into 242 the Appendix.

${ }_{243}$ Claim $1\left|\mathcal{S}_{\min }^{\theta^{*}}(v)\right| \geq 2$.

Taking into account this claim, define $\alpha \in \mathbb{R}^{N}$ as

$$
\alpha_{i}= \begin{cases}\ell_{i}^{\theta^{*}}(v)-\varepsilon & \text { if } i=i_{t^{*}} \\ \ell_{i}^{\theta^{*}}(v) \quad \text { if } i \in N, i \neq i_{t^{*}}\end{cases}
$$

where

$$
\begin{gathered}
0<\varepsilon<\min \left\{\ell^{\theta^{*}}(v)(M)-v(M)\right\} . \\
M \subseteq N \\
\ell^{\theta^{*}}(v)(M)>v(M)
\end{gathered}
$$

Notice that the parameter $\varepsilon$ is well defined since (11) holds. Moreover, we have

Take $I^{\theta^{*}}=\bigcap_{M \in \mathcal{S}_{\text {min }}^{\theta^{*}}(v)} M$ and notice that, by definition, $i_{t^{*}} \in I^{\theta^{*}}$ (and thus 248 $\left.I^{\theta^{*}} \neq \varnothing\right), M \backslash I^{\theta^{*}} \neq \varnothing$ for all $M \in \mathcal{S}_{\text {min }}^{\theta^{*}}(v)$, and $N \backslash I^{\theta^{*}} \neq \varnothing$ where the last two 249 assertions follow from Claim 1. 
Then define the game $\left(N \backslash I^{\theta^{*}}, \omega\right)$ as follows:

$$
\begin{aligned}
\omega(\varnothing)= & 0, \\
\omega(R)= & \max \left\{v\left(R^{\prime} \cup Q\right)-\alpha\left(R^{\prime} \cup Q\right)\right\} \text { for all } \varnothing \neq R \subseteq N \backslash I^{\theta^{*}} . \\
& R^{\prime} \subseteq R \\
& Q \subseteq I^{\theta^{*}}
\end{aligned}
$$

Let us remark that $\omega(R) \in\{0, \varepsilon\}$, for all $R \subseteq N \backslash I^{\theta^{*}}$, and $\omega\left(N \backslash I^{\theta^{*}}\right)=\varepsilon$. To check this we first claim that, given $M \subseteq N$, we have

$$
v(M)-\alpha(M)= \begin{cases}0 & \text { if } M=\varnothing \\ \varepsilon & \text { if } M \in \mathcal{S}^{\theta^{*}}(v) \text { or } M=N \\ \leq 0 \text { otherwise. }\end{cases}
$$

252 Indeed, if $M=\varnothing, v(\varnothing)-\alpha(\varnothing)=0$; if $M \in \mathcal{S}^{\theta^{*}}(v)$ or $M=N$ then $v(M)=$ $\ell^{\theta^{*}}(v)(M)$ and $v(M)-\alpha(M)=v(M)-\ell^{\theta^{*}}(v)(M)+\varepsilon=\varepsilon$; finally, if $M \notin \mathcal{S}^{\theta^{*}}(v)$ and $M \neq N$, $\varnothing$ then either $i_{t^{*}} \notin M$ or $v(M)<\ell^{\theta^{*}}(v)(M)$ : if $i_{t^{*}} \notin M$, then $v(M)-$ $\alpha(M)=v(M)-\ell^{\theta^{*}}(v)(M) \leq 0$, and if $i_{t^{*}} \in M$ but $v(M)<\ell^{\theta^{*}}(v)(M)$, then $256 v(M)-\alpha(M)=v(M)-\ell^{\theta^{*}}(v)(M)+\varepsilon<0$, where the last strict inequality follows 257 from the definition of $\varepsilon$.

Taking (12) into account, and since for all $R \subseteq N \backslash I^{\theta^{*}}, \omega(R)$ is the maximum of differences $v(M)-\alpha(M)$, where $M \subseteq R \cup I^{\theta^{*}}$, it follows that $\omega(R) \in\{0, \varepsilon\}$. To 260 see $\omega\left(N \backslash I^{\theta^{*}}\right)=\varepsilon$ just take $R^{\prime}=N \backslash I^{\theta^{*}}$ and $Q=I^{\theta^{*}}$ in its definition.

Now take an element $\delta \in \mathbb{R}^{N \backslash I^{\theta^{*}}}$ in the prekernel of the game $\left(N \backslash I^{\theta^{*}}, \omega\right)$, that is $\delta \in \mathcal{P} \mathcal{K}\left(N \backslash I^{\theta^{*}}, \omega\right)$. By the monotonicity of the game $\left(N \backslash I^{\theta^{*}}, \omega\right)$ and Theorem 
5.6.1 in Peleg and Südholter (2007) it follows ${ }^{5}$ that

(a) $\delta_{i} \geq 0$, for all $i \in N \backslash I^{\theta^{*}}$.

(b) $\delta_{i}=0$, for all $i \in N \backslash \bigcup_{M \in \mathcal{S}_{\text {min }}^{\theta^{*}}(v)} M$.

To see (b), let $i \in N \backslash \bigcup_{M \in \mathcal{S}_{\text {min }}^{\theta^{*}}(v)} M$ and let $R \subseteq N \backslash I^{\theta^{*}}$ with $i \in R$. Let us check that $\omega(R)-\omega(R \backslash\{i\})=0$. If $\omega(R)=0$, by the monotonicity of the game $\omega$, we are done. If $\omega(R)=\varepsilon$, we know by (12) that $\omega(R)=v(M)-\alpha(M)$, for some coalition $M \in \mathcal{S}^{\theta^{*}}(v)$ or $\omega(R)=v(N)-\alpha(N)$. In case $\omega(R)=v(M)-\alpha(M)$ for some $M \in \mathcal{S}^{\theta^{*}}(v)$, we can take, in fact, $M \in \mathcal{S}_{\text {min }}^{\theta^{*}}(v)$; in case $\omega(R)=v(N)-\alpha(N)$, then $R=N \backslash I^{\theta^{*}}$ and for any $M \in S_{\min }^{\theta^{*}}(v)$ we have that $\varepsilon=w\left(N \backslash I^{\theta^{*}}\right) \geq w\left(M \backslash I^{\theta^{*}}\right) \geq$ $v(M)-\alpha(M)=\varepsilon$, where the first inequality follows from the monotonicity of the game and the last equality by (12). Thus we conclude that, in any of both cases, $\omega(R)=v(M)-\alpha(M)$ with $M \in S_{\min }^{\theta^{*}}(v)$. Now, since by hypothesis of case (b) player $i$ does not belong to any minimal coalition in $\mathcal{S}_{m i n}^{\theta^{*}}(v)$, it follows $M \subseteq(R \backslash\{i\}) \cup I^{\theta^{*}}$ and then we conclude that

$$
\begin{gathered}
\varepsilon \geq \omega(R \backslash\{i\})=\max _{\substack{R^{\prime} \subseteq R \backslash\{i\} \\
Q \subseteq I^{\theta^{*}}}}\left\{v\left(R^{\prime} \cup Q\right)-\alpha\left(R^{\prime} \cup Q\right)\right\} \geq v(M)-\alpha(M)=\varepsilon,
\end{gathered}
$$

261 and we are done.

Next define the vector $x \in \mathbb{R}^{N}$ as follows:

$$
x_{i}=\left\{\begin{array}{l}
\alpha_{i}+\delta_{i} \text { if } i \in N \backslash I^{\theta^{*}} \\
\alpha_{i} \quad \text { if } i \in I^{\theta^{*}} .
\end{array}\right.
$$

5 We are using the fact that for any $\delta \in \mathcal{P K}\left(N \backslash I^{\theta^{*}}, \omega\right)$ and for any $i \in N \backslash I^{\theta^{*}}$,

$$
\min _{R \subseteq N \backslash I^{\theta^{*}}: i \in R}\{\omega(R)-\omega(R \backslash\{i\})\} \leq \delta_{i} \leq \max _{R \subseteq N \backslash I^{\theta^{*}}: i \in R}\{\omega(R)-\omega(R \backslash\{i\})\} .
$$


At this point, it is also important to notice that $\ell_{i_{t^{*}}}^{\theta^{*}}-\varepsilon \geq v\left(\left\{i_{t^{*}}\right\}\right)$. Thus, $x$ is an imputation of the game $(N, v)$. $x \notin C(N, v)$

The vector $x$ is efficient, $x(N)=v(N)$, and individually rational in the original game $(N, v)$, i.e. $x_{i} \geq v(\{i\})$, for all $i \in N$. Only the case $i=i_{t^{*}}$ deserves some attention. Indeed, by $(9)$, we have $\ell_{i_{t^{*}}}^{\theta^{*}}(v)>m_{i_{t^{*}}}^{\theta^{*}}(v) \geq v\left(\left\{i_{t^{*}}\right\}\right)$, where the last inequality comes by zero-monotonicity of the game $(N, v)$. Therefore, $x_{i_{t^{*}}}=\alpha_{i_{t^{*}}}=$

However, let us argue that $x \notin C(N, v)$. As $\delta\left(N \backslash I^{\theta^{*}}\right)=\varepsilon>0$, there must exist $i \in N \backslash I^{\theta^{*}}$ such that $\delta_{i}>0$. Moreover, there also must exist $M \in \mathcal{S}_{\min }^{\theta^{*}}(v)$ such that $i \notin M$ (otherwise $\left.i \in I^{\theta^{*}}\right)$. Hence, $\delta\left(M \backslash I^{\theta^{*}}\right)<\varepsilon$ and thus $v(M)-x(M)=$ $v(M)-\ell^{\theta^{*}}(v)(M)+\varepsilon-\delta\left(M \backslash I^{\theta^{*}}\right)>v(M)-\ell^{\theta^{*}}(v)(M)=0$, where the last equality comes from $M \in \mathcal{S}_{\text {min }}^{\theta^{*}}(v)$. Hence, we obtain that $v(M)-x(M)>0$ and we conclude

To see this, if $M \notin \mathcal{S}^{\theta^{*}}(v)$ then, by $(12), v(M)-\alpha(M) \leq 0$. Hence, $v(M)-x(M)=$ $v(M)-\alpha(M)-\delta\left(M \backslash I^{\theta^{*}}\right) \leq 0$, which involves a contradiction.

We finally check that $x$ is in the steady bargaining set of the game $v$. To this aim take $S \subseteq N$ such that $v(S)-x(S)>0$. We shall prove there exists $M \subseteq N$ such that $M \backslash S \neq \varnothing, S \backslash M \neq \varnothing, S \cap M \neq \varnothing$ and $v(M)-x(M) \geq v(S)-x(S)$.

First, recall that $S \in \mathcal{S}^{\theta^{*}}(v)$ (see (15)). Now, let $S^{\prime} \in \mathcal{S}_{\text {min }}^{\theta^{*}}(v)$ with $S^{\prime} \subseteq S$ such that

$$
v\left(S^{\prime}\right)-x\left(S^{\prime}\right) \geq v(P)-x(P), \text { for all } P \in \mathcal{S}_{\min }^{\theta^{*}}(v) \text { with } P \subseteq S .
$$

Since $v(S)-x(S)=v(S)-\alpha(S)-\delta\left(S \backslash I^{\theta^{*}}\right)=v(S)-\ell^{\theta^{*}}(v)(S)+\varepsilon-\delta\left(S \backslash I^{\theta^{*}}\right)=$ $\varepsilon-\delta\left(S \backslash I^{\theta^{*}}\right)>0$, we have $\delta\left(S \backslash I^{\theta^{*}}\right)<\varepsilon$. Therefore, there must exist $j \in N \backslash I^{\theta^{*}}$, 

$S^{\prime} \subseteq S$ at $x$. Taking this fact into account we also have

$$
\begin{aligned}
0<v(S)-x(S) & \leq v\left(S^{\prime}\right)-x\left(S^{\prime}\right)=v\left(S^{\prime}\right)-\alpha\left(S^{\prime}\right)-\delta\left(S^{\prime} \backslash I^{\theta^{*}}\right) \\
& \leq \omega\left(S^{\prime} \backslash I^{\theta^{*}}\right)-\delta\left(S^{\prime} \backslash I^{\theta^{*}}\right) \\
& \leq s_{i j}^{\omega}(\delta)=s_{j i}^{\omega}(\delta)=\omega(R)-\delta(R),
\end{aligned}
$$

288 for some $R \subseteq N \backslash I^{\theta^{*}}$ such that $j \in R$ but $i \notin R$. Finally,

$$
\begin{aligned}
\omega(R)-\delta(R) & =v\left(R^{\prime} \cup Q\right)-\alpha\left(R^{\prime} \cup Q\right)-\delta(R) \\
& \leq v\left(R^{\prime} \cup Q\right)-\alpha\left(R^{\prime} \cup Q\right)-\delta\left(R^{\prime}\right)=v\left(R^{\prime} \cup Q\right)-x\left(R^{\prime} \cup Q\right),
\end{aligned}
$$

289 for some $R^{\prime} \subseteq R$ and $Q \subseteq I^{\theta^{*}}$. The coalition $M=R^{\prime} \cup Q$ is precisely the one we since $i \notin R \cup I^{\theta^{*}}$.

First, from (18) and (19), we can deduce that $v(S)-x(S) \leq v(M)-x(M)$. Furthermore, since $v(M)-x(M)>0$ and $v(S)-x(S)>0$, by (15), it follows that $I^{\theta^{*}} \subseteq M \cap S$ which implies $M \cap S \neq \varnothing$. Moreover, $i \in S \backslash M$ since $i \in S^{\prime} \subseteq S$ and $i \notin R^{\prime}($ since $i \notin R)$ and $i \notin Q\left(\right.$ since $\left.i \notin I^{\theta^{*}}\right)$.

Finally, if $j \in M$ then $j \in M \backslash S$ and thus $M \backslash S \neq \varnothing$. If $j \notin M$, we still claim that $M \backslash S \neq \varnothing$. To check it, let us suppose on the contrary that $M \subseteq S$ and $j \notin M$.

Taking into account that $\delta_{j}>0, j \in R$ and $j \notin M$ the non-strict inequality in (19) 

obtain

$$
0<v\left(S^{\prime}\right)-x\left(S^{\prime}\right)<v(M)-x(M)
$$

By (15), there exists $M^{\prime} \subseteq M$ where $M^{\prime} \in \mathcal{S}_{\min }^{\theta^{*}}(v)$. Therefore,

$$
v(M)-x(M)=\varepsilon-\delta\left(M \backslash I^{\theta^{*}}\right) \leq v\left(M^{\prime}\right)-\alpha\left(M^{\prime}\right)-\delta\left(M^{\prime} \backslash I^{\theta^{*}}\right)=v\left(M^{\prime}\right)-x\left(M^{\prime}\right) .
$$

By (20) and (21) we conclude that $v\left(S^{\prime}\right)-x\left(S^{\prime}\right)<v\left(M^{\prime}\right)-x\left(M^{\prime}\right)$ being $M^{\prime} \in$ $\mathcal{S}_{\min }^{\theta^{*}}(v)$ and $M^{\prime} \subseteq M \subseteq S$. However, this contradicts (16) and we conclude that $M \backslash S \neq \varnothing$. This last result proves that $x$ is not in the core of $v$ but in its steady bargaining set, and the proof of this implication ends.

As far as we know the conditions of zero-monotonicity and gran coalition superadditivity cannot be dropped from Theorem 1 out.

Concerning the zero-monotonicity condition, next example proves its necessity. Let $(N, v)$ be a four-player game where $N=\{1,2,3,4\}$ and $v(S)=1$, if $|S|=1$, $v(S)=2$, if $|S|=2$ or $|S|=3$ and $v(N)=4$. It is easy to see that $(N, v)$ is neither convex nor zero-monotonic, but $C(N, v)=\mathcal{S B}(N, v)=\{(1,1,1,1)\}$.

Grand coalition superadditivity is needed to guarantee that the steady bargaining set is nonempty. It remains an open question whether zero-monotonicity implies the nonemptiness of the steady bargaining set.

As a consequence of Theorem 1 and the characterization result of Izquierdo and Rafels (2012) we get the following corollary.

Corollary 1 Let $v \in \mathcal{G}^{N}$ be a zero-monotonic and grand coalitional superadditive game. Then, the following statements are equivalent:

1. $\mathcal{Z}^{*}(N, v)=C(N, v)$.

2. $v$ is a convex game. 


\section{Conclusions}

Bargaining sets face the problem of distributing profits focusing on the negotiation (objections and counterobjections) between agents. Besides this, there are concepts of bargaining sets (e.g. Davis and Maschler $(1963,1967)$ or Shubik (1984)) that put the stress on the player who leads the objection. For these bargaining sets, there are examples of non-convex cooperative games for whom the core and the bargaining set do coincide (for instance, this is the case of average monotonic cooperative games (Izquierdo and Rafels, 2001), or assignment games (for the proof of this coincidence see Solymosi(2008)).

In Izquierdo and Rafels (2012), it has been already shown that a modification of the Mas-Colell bargaining set (Shimomura 1997) has been useful to characterize the convexitiy of a game This notion of bargaining set considers objections and counterobjections as proposals made by a group rather than an action led by an specific player. It is also important to remark that agents receive strictly better rewards in objections and counterobjections. Following this idea of group proposals and strictly positive incentives, we have proved in this paper that the modified Zhou bargaining set also characterizes convex games within the class of zero-monotonic and grand coalition superadditive games. The difference between both bargaining sets relies on the qualification of coalitions than might counterobject: while in the Mas-Colell version there are no restrictions on which are the coalitions $T$ that are allowed to react to an objection made by a coalition $S$, the Zhou's framework requires some conditions. First, there must be at least one player 339 belonging to both coalitions; if not, $S \cap T=\varnothing$, and the counterobjection might be interpreted as a different objection rather than a proper counter-objection. 
Second, at least one player involved in the objection must not be involved in the counterobjection; if not, $S \subseteq T$, and the counterobjection might be interpreted as a reinforcement to the objection. Finally, the counterobjecting coalition must involved at least an agent not taking part in the objection; if not, $T \subseteq S$, but this fact might suggest that the original objection should be revised but not rejected.

From the point of view of characterizing convex games, our result reveals that it is not so important if we just consider one, two, three or none of the above requirements for the counterobjecting coalitions. Objections and counter-objections made as a group and strictly positive incentives are the important keys to reach these results.

\section{References}

1. Davis M, Maschler M (1963) Existence of stable payoff configurations for cooperative games. Bull. Am. Math. Soc. 69: 106-108

2. Davis M, Maschler M (1965) The kernel of a cooperative game. Nav Res Logist Quart 2: $223-259$

3. Davis M, Maschler M (1967) Existence of stable payoff configurations for cooperative games. In: Shubik M(ed) Essays in mathematical economics in honour of Oskar Morgenstern. Princeton University Press, Princeton, pp 39-52.

4. Einy E, Wettstein D (1996) Equivalence between bargaining sets and the core in simple games. Internat. J Game Theory 25: 65-71

5. Greenberg J (1992) On the sensitivity of von Neumann and Morgenstern abstract stable sets: The stable and the individual stable bargaining set. Internat. J Game Theory 21: $41-55$

6. Ichiishi T (1981) Super-modularity: applications to convex games and to the greedy algorithm in LP. J Econ Theory 25: 283-286

7. Izquierdo JM, Rafels C (2001) Average monotonic cooperative games. Games Econ Behav 36(2): 174-192 
8. Izquierdo JM, Rafels C (2012) A characterization of convex TU games by means of the Mas-Colell bargaining set ( à la Shimomura). Int J Game Theory 41(2): 381-395

9. Kikuta K (1997) The kernel for reasonable outcomes in a cooperative game. Int J Game Theory 26: 51-59

10. Mas-Colell A (1989) An equivalence theorem for a bargaining set. J Math Econ 18: 129-139

11. Núñez M, Rafels C (1998) On extreme points of the core and reduced games. Ann Oper Research 84: 121-133

12. Peleg B, Sudhölter P (2007) Introduction to the Theory of Cooperative Games. Kluwer Academic Publishers, Dordrecht, The Netherlands (2nd edition)

13. Schmeidler D (1969) The nucleolus of a characteristic function game. SIAM J Appl Math 17(6): 1163-1170

14. Shapley LS (1971) Cores of convex games. Int J Game Theory 1(3): 11-26

15. Shapley LS, Shubik M (1966) Quasi-cores in a monetary economy with non-convex preferences, Econometrica 34(4): 805-827

16. Sharkey WW (1982) Cooperative games with large cores. Int J Game Theory 11(3): 175182

17. Shimomura K (1997) Quasi-Cores in Bargaining Sets. Int J Game Theory 26: 283-302

18. Shubik M (1984) A Game Theoretic Approach to Political Economy. MIT Press, Cambridge, Massachusetts.

19. Solymosi T (2008) Bargaining sets and the core in partioning games. Central European J Oper Res 16(4): 425-440.

20. Zhou L (1994) A new bargaining set of an n-person game and endogenous coalition formation. Games Econ Behav 6: 512-526

\section{Appendix}

\section{Proof of Claim 1.}

Let us recall that the claim is under the hypothesis $C(N, v)=\mathcal{S B}(N, v)$. Next, assume $\left|\mathcal{S}_{\text {min }}^{\theta^{*}}(v)\right|=1$, say $\mathcal{S}_{\text {min }}^{\theta^{*}}(v)=\left\{S^{*}\right\}$, where $\theta^{*}=\left(i_{1}, i_{2}, \ldots, i_{n}\right)$. Then, we 
Proof Let us suppose that there exists $i_{t^{\prime}} \in S^{*}$ with $t^{\prime} \in\left\{t^{*}+1, t^{*}+2, \ldots, n\right\}$. Then, define the vector $x \in \mathbb{R}^{N}$ as $x_{i_{t^{*}}}=\ell_{i_{t^{*}}}^{\theta^{*}}(v)-\varepsilon_{2}, x_{i_{t^{\prime}}}=\ell_{i_{t^{\prime}}}^{\theta^{*}}(v)+\varepsilon_{2}$ and $x_{i_{k}}=\ell_{i_{k}}^{\theta^{*}}(v)$, else, where

$$
\begin{gathered}
0<\varepsilon_{2}<\min \left\{\ell^{\theta^{*}}(v)(M)-v(M)\right\} . \\
i_{t^{*}} \in M \subseteq N \\
\ell^{\theta^{*}}(v)(M)>v(M)
\end{gathered}
$$

By (11), the parameter $\varepsilon_{2}$ is well-defined. To end the proof, we show that $x \in$ $C(N, v)$ contradicting $\ell^{\theta^{*}}(v)$ to be the lexmin core vector of $v$ relative to $\theta^{*}$. To see this point, it is straightforward that $x(N)=v(N)$. Moreover, if $M \subseteq N, M \neq N$, and $i_{t^{*}} \notin M$ then $x(M) \geq \ell^{\theta^{*}}(v)(M) \geq v(M)$. If $i_{t^{*}} \in M$ and $\ell^{\theta^{*}}(v)(M)>v(M)$, then $x(M) \geq \ell^{\theta^{*}}(v)(M)-\varepsilon_{2}>\ell^{\theta^{*}}(v)(M)-\left(\ell^{\theta^{*}}(v)(M)-v(M)\right)=v(M)$. Finally, if $i_{t^{*}} \in M$ and $\ell^{\theta^{*}}(v)(M)=v(M)$ then $M \in \mathcal{S}^{\theta}(v)$, and $S^{*} \subseteq M$ since there is a unique minimal coalition in $\mathcal{S}^{\theta^{*}}(v)$. Thus $i_{t^{\prime}} \in M$. Hence, $x(M)=\ell^{\theta^{*}}(v)(M) \geq v(M)$ and $x \in C(N, v)$, ending the proof of this subclaim.

Subclaim 1.2 The number of players in $T^{*}=\left\{i_{1}, \ldots, i_{t^{*}}\right\}$ is at least three, i.e. $t^{*} \geq$ 3.

Proof It is clear that if $t^{*}=1$ then $T^{*}=\left\{i_{1}\right\}$ and the unique minimal coalition in $\mathcal{S}^{\theta^{*}}(v)$ must be $S^{*}=\left\{i_{1}\right\}$. Then, by $(9), \ell_{i_{1}}^{\theta^{*}}(v)>m_{i_{1}}^{\theta^{*}}(v)=v\left(\left\{i_{1}\right\}\right)$ which contradicts $S^{*} \in \mathcal{S}^{\theta^{*}}(v)$. Moreover if $t^{*}=2$ then $T^{*}=\left\{i_{1}, i_{2}\right\}, \ell_{i_{1}}^{\theta^{*}}(v)=m_{i_{1}}^{\theta^{*}}(v)=$ $v\left(\left\{i_{1}\right\}\right)$ by $(8)$, and $\ell_{i_{2}}^{\theta^{*}}(v)>m_{i_{2}}^{\theta^{*}}(v)=v\left(\left\{i_{1}, i_{2}\right\}\right)-v\left(\left\{i_{1}\right\}\right) \geq v\left(\left\{i_{2}\right\}\right)$, where the last 
inequality comes from zero-monotonicity. From this we deduce $\ell_{i_{1}}^{\theta^{*}}(v)+\ell_{i_{2}}^{\theta^{*}}(v)>$ $v\left(\left\{i_{1}, i_{2}\right\}\right)$, which contradicts the fact that the unique minimal coalition in $\mathcal{S}^{\theta^{*}}(v)$ must be a subset of $T^{*}$ (see Subclaim 1.1.).

Let us recall (see $(10))$ that any subgame $\left(R, v_{R}\right)$, with $R \subseteq T^{*}, R \neq T^{*}$, is a convex game. Therefore, the maximal marginal contribution of player $i_{t^{*}} \in N$ to any subcoalition ${ }^{6} Q \varsubsetneqq T^{*} \backslash\left\{i_{t^{*}}\right\}$ is attained at a coalition containing $t^{*}-2$ players; that is, without loss of generality $\max _{Q \varsubsetneqq T^{*} \backslash\left\{i_{t^{*}}\right\}}\left\{v\left(Q \cup\left\{i_{t^{*}}\right\}\right)-v(Q)\right\}=v\left(\left\{i_{1}, i_{2}, \ldots, i_{t^{*}-2}, i_{t^{*}}\right\}\right)-v\left(\left\{i_{1}, i_{2}, \ldots, i_{t^{*}-2}\right\}\right)$.

Subclaim $1.3 \ell_{i_{t^{*}}}^{\theta^{*}}(v)=v\left(\left\{i_{1}, i_{2}, \ldots, i_{t^{*}-2}, i_{t^{*}}\right\}\right)-v\left(\left\{i_{1}, i_{2}, \ldots, i_{t^{*}-2}\right\}\right)$.

Proof First, by (8), if $\ell_{i_{t^{*}}}^{\theta^{*}}(v)<v\left(\left\{i_{1}, i_{2}, \ldots, i_{t^{*}-2}, i_{t^{*}}\right\}\right)-v\left(\left\{i_{1}, i_{2}, \ldots, i_{t^{*}-2}\right\}\right)=$ $v\left(\left\{i_{1}, i_{2}, \ldots, i_{t^{*}-2}, i_{t^{*}}\right\}\right)-\ell^{\theta^{*}}(v)\left(\left\{i_{1}, i_{2}, \ldots, i_{t^{*}-2}\right\}\right)$, then

$$
\ell^{\theta^{*}}(v)\left(\left\{i_{1}, i_{2}, \ldots, i_{t^{*}-2}, i_{t^{*}}\right\}\right)<v\left(\left\{i_{1}, i_{2}, \ldots, i_{t^{*}-2}, i_{t^{*}}\right\}\right)
$$

402 which contradicts $\ell^{\theta^{*}}(v) \in C(N, v)$.

On the other hand, if $\ell_{i_{t^{*}}}^{\theta^{*}}(v)>v\left(\left\{i_{1}, i_{2}, \ldots, i_{t^{*}-2}, i_{t^{*}}\right\}\right)-v\left(\left\{i_{1}, i_{2}, \ldots, i_{t^{*}-2}\right\}\right)$, then, since $\ell^{\theta^{*}}(v) \in C(N, v)$,

$$
\begin{aligned}
\ell_{i_{t^{*}}}^{\theta^{*}}(v) & >v\left(\left\{i_{1}, i_{2}, \ldots, i_{t^{*}-2}, i_{t^{*}}\right\}\right)-v\left(\left\{i_{1}, i_{2}, \ldots, i_{t^{*}-2}\right\}\right) \\
& =\max _{Q \nsubseteq T^{*} \backslash\left\{i_{t^{*}}\right\}}\left\{v\left(Q \cup\left\{i_{t^{*}}\right\}\right)-v(Q)\right\} \geq \max _{Q \mp T^{*} \backslash\left\{i_{t^{*}}\right\}}\left\{v\left(Q \cup\left\{i_{t^{*}}\right\}\right)-\ell^{\theta^{*}}(v)(Q)\right\} .
\end{aligned}
$$

Thus, $\ell^{\theta^{*}}(v)\left(Q \cup\left\{i_{t^{*}}\right\}\right)>v\left(Q \cup\left\{i_{t^{*}}\right\}\right)$, for all $Q \varsubsetneqq\left\{i_{1}, i_{2}, \ldots, i_{t^{*}-1}\right\}$. However adding this result to ( 11 ) we reach a contradiction with Subclaim 1.1.

Next, let us define

$$
J^{\theta^{*}}=\left\{i \in T^{*}=\left\{i_{1}, i_{2}, \ldots, i_{t^{*}}\right\} \mid \ell^{\theta^{*}}(v)\left(T^{*} \backslash\{i\}\right)=v\left(T^{*} \backslash\{i\}\right)\right\} .
$$

${ }^{6}$ The symbol $\varsubsetneqq$ between two coalitions $S \varsubsetneqq T$ means $S \subseteq T$ and $S \neq T$. 
403

${ }_{405}\left\{i_{t^{*}-1}, i_{t^{*}}\right\} \subseteq J^{\theta^{*}}$. Therefore,

$$
\left|J^{\theta^{*}}\right| \geq 2
$$

Finally, by zero-monotonicity of the game $v$, it holds

$$
\ell_{i}^{\theta^{*}}(v)>v(\{i\}), \text { for all } i \in J^{\theta^{*}} .
$$

$$
\begin{aligned}
v\left(T^{*} \backslash\left\{i_{\kappa}\right\}\right) & \leq \ell^{\theta^{*}}(v)\left(T^{*} \backslash\left\{i_{\kappa}\right\}\right)=\ell^{\theta^{*}}(v)\left(T^{*} \backslash\left(S \cup\left\{i_{\kappa}\right\}\right)\right)+\ell^{\theta^{*}}(v)(S) \\
& =m^{\theta^{*}}(v)\left(T^{*} \backslash\left(S \cup\left\{i_{\kappa}\right\}\right)\right)+\ell^{\theta^{*}}(v)(S)
\end{aligned}
$$

Notice that $T^{*} \backslash\left(S \cup\left\{i_{\kappa}\right\}\right) \subseteq\left\{i_{1}, i_{2}, \ldots, i_{\kappa-1}\right\}$ and thus we describe $T^{*} \backslash(S \cup$ $\left.\left\{i_{\kappa}\right\}\right)=\left\{i_{r_{1}}, i_{r_{2}}, \ldots, i_{r_{m}}\right\}$, where $r_{1}<r_{2}<\cdots<r_{m}<\kappa$. Moreover, and for all $i_{r_{j}} \in T^{*} \backslash\left(S \cup\left\{i_{\kappa}\right\}\right)$, let us denote by $P_{i_{r_{j}}}^{\theta^{*}}=\left\{i_{1}, i_{2}, \ldots, i_{\left(r_{j}\right)-1}\right\} \subseteq N$ the set of predecessors of player $i_{r_{j}}$ relative to the ordering

$$
\theta^{*}=\left(i_{1}, i_{2}, \ldots, i_{r_{1}}, \ldots, i_{r_{2}}, \ldots, i_{r_{j}}, \ldots, i_{\kappa}, i_{\kappa+1}, \ldots, i_{n}\right)
$$


Then, we have

$$
\begin{aligned}
& m^{\theta^{*}}(v)\left(T^{*} \backslash\left(S \cup\left\{i_{\kappa}\right\}\right)\right)=\sum_{j=1}^{m} m_{r_{j}}^{\theta^{*}}(v)=\sum_{j=1}^{m}\left[v\left(P_{i_{r_{j}}}^{\theta^{*}} \cup\left\{i_{r_{j}}\right\}\right)-v\left(P_{i_{r_{j}}}^{\theta^{*}}\right)\right] \\
& \leq \sum_{j=1}^{m-1}\left[v\left(\left(P_{i_{r_{m}}}^{\theta^{*}} \backslash\left\{i_{r_{j}}, i_{r_{j+1}}, \ldots, i_{r_{m-1}}\right\}\right) \cup\left\{i_{r_{j}}\right\}\right)-v\left(P_{i_{r_{m}}}^{\theta^{*}} \backslash\left\{i_{r_{j}}, i_{r_{j+1}}, \ldots, i_{r_{m-1}}\right\}\right)\right] \\
& \quad+v\left(P_{i_{r_{m}}}^{\theta^{*}} \cup\left\{i_{r_{m}}\right\}\right)-v\left(P_{i_{r_{m}}}^{\theta^{*}}\right) \\
& =v\left(P_{i_{r_{m}}}^{\theta^{*}} \cup\left\{i_{r_{m}}\right\}\right)-v\left(P_{i_{r_{m}}}^{\theta^{*}} \backslash\left\{i_{r_{1}}, i_{r_{2}}, \ldots, i_{r_{m-1}}\right\}\right) \\
& \leq v\left(P_{i_{r_{m}}}^{\theta^{*}} \cup\left\{i_{t^{*}}\right\} \cup\left\{i_{r_{m}}\right\}\right)-v\left(\left(P_{i_{r_{m}}}^{\theta^{*}} \cup\left\{i_{t^{*}}\right\}\right) \backslash\left\{i_{r_{1}}, i_{r_{2}}, \ldots, i_{r_{m-1}}\right\}\right) \\
& \leq v\left(T^{*} \backslash\left\{i_{\kappa}\right\}\right)-v\left(T^{*} \backslash\left\{i_{r_{1}}, i_{r_{2}}, \ldots, i_{r_{m}}, i_{\kappa}\right\}\right) \\
& =v\left(T^{*} \backslash\{i \kappa\}\right)-v(S),
\end{aligned}
$$

where the first inequality follows from (2), the convexity of the subgame $\left(T^{*} \backslash\left\{i_{\kappa}\right\}, v_{T^{*} \backslash\left\{i_{\kappa}\right\}}\right)$ and the fact that, for all $j=1, \ldots, m-1$, we have

$$
P_{i_{r_{j}}}^{\theta^{*}} \subseteq P_{i_{r_{m}}}^{\theta^{*}} \backslash\left\{i_{r_{j}}, i_{r_{j+1}}, \ldots, i_{r_{m-1}}\right\}
$$

${ }_{415}$ the second inequality follows from the convexity of the subgame $\left(T^{*} \backslash\left\{i_{\kappa}\right\}, v_{T^{*}} \backslash\{i \kappa\}\right)$, ${ }_{416}$ and the third one by taking in (3) $M=P_{i_{r_{m}}}^{\theta^{*}} \cup\left\{i_{t^{*}}\right\} \cup\left\{i_{r_{m}}\right\}$ and $M^{\prime}=T^{*} \backslash$

${ }_{417}\left\{i_{r_{1}}, i_{r_{2}}, \ldots, i_{r_{m}}, i_{\kappa}\right\}$. Therefore, we obtain that $m^{\theta^{*}}(v)\left(T^{*} \backslash\left(S \cup\left\{i_{\kappa}\right\}\right)\right) \leq v\left(T^{*} \backslash\right.$

$\left.{ }_{418}\left\{i_{\kappa}\right\}\right)-v(S)$. Using this inequality in (24) we obtain

$$
\begin{aligned}
v\left(T^{*} \backslash\left\{i_{\kappa}\right\}\right) & \leq \ell^{\theta^{*}}(v)\left(T^{*} \backslash\left\{i_{\kappa}\right\}\right) \leq m^{\theta^{*}}(v)\left(T^{*} \backslash\left(S \cup\left\{i_{\kappa}\right\}\right)\right)+\ell^{\theta^{*}}(v)(S) \\
& \leq v\left(T^{*} \backslash\left\{i_{\kappa}\right\}\right)-v(S)+\ell^{\theta^{*}}(v)(S)=v\left(T^{*} \backslash\left\{i_{\kappa}\right\}\right) .
\end{aligned}
$$

Therefore, we conclude that $v\left(T^{*} \backslash\left\{i_{\kappa}\right\}\right)=\ell^{\theta^{*}}(v)\left(T^{*} \backslash\left\{i_{\kappa}\right\}\right)$ which implies $i_{\kappa} \in J^{\theta^{*}} \backslash S$, as we want to prove.

${ }_{419}$ Once we have proved the above subclaims, let us define the vector $\beta \in \mathbb{R}^{N}$ as

$$
\beta_{i}= \begin{cases}\ell_{i}^{\theta^{*}}(v)-\varepsilon_{3} & \text { if } i \in J^{\theta^{*}} \\ \ell_{i}^{\theta^{*}}(v) & \text { if } i \in N \backslash J^{\theta^{*}}\end{cases}
$$


where

$$
\begin{gathered}
0<n \cdot \varepsilon_{3}<\min _{M \subseteq N}\left\{\ell^{\theta^{*}}(v)(M)-v(M)\right\} . \\
\ell^{\theta^{*}}(v)(M)>v(M)
\end{gathered}
$$

420 By $(23)$,

$$
\beta_{i} \geq v(\{i\}), \text { for all } i \in J^{\theta^{*}}
$$

421 Hence, define the game $\left(N \backslash T^{*}, \omega\right)$ as follows:

$$
\begin{aligned}
& \omega(\varnothing)=0 \\
& \omega(R)=\max _{Q \subseteq T^{*}}\{v(R \cup Q)-\beta(R \cup Q)\}, \text { for all } \varnothing \neq R \subseteq N \backslash T^{*} .
\end{aligned}
$$

422 Let us remark that $\omega(R) \leq\left|J^{\theta^{*}}\right| \cdot \varepsilon_{3}$, for any $\varnothing \neq R \subseteq N \backslash T^{*}$. To check it, 423 simply notice that $\omega(R)=v\left(R \cup Q^{*}\right)-\beta\left(R \cup Q^{*}\right)$ for some $Q^{*} \subseteq T^{*}$, and thus $\omega(R)=v\left(R \cup Q^{*}\right)-\beta\left(R \cup Q^{*}\right)=v\left(R \cup Q^{*}\right)-\ell^{\theta^{*}}\left(R \cup Q^{*}\right)+\left|Q^{*} \cap J^{\theta^{*}}\right| \cdot \varepsilon_{3} \leq \mid Q^{*} \cap$ $J^{\theta^{*}}\left|\cdot \varepsilon_{3} \leq\right| J^{\theta^{*}} \mid \cdot \varepsilon_{3}$. Moreover, for the case $R=N \backslash T^{*}$ we have $\omega\left(N \backslash T^{*}\right)=\left|J^{\theta^{*}}\right| \cdot \varepsilon_{3}$, just by taking $Q=T^{*}$ in its definition.

Next, define the subset $Y$ of vectors in $\mathbb{R}^{N \backslash T^{*}}$ as follows:

$Y=\left\{\alpha \in \mathbb{R}^{N \backslash T^{*}} \mid \alpha_{i} \geq 0\right.$, for all $i \in N \backslash T^{*}$ and $\left.\alpha\left(N \backslash T^{*}\right)=\omega\left(N \backslash T^{*}\right)=\left|J^{\theta^{*}}\right| \cdot \varepsilon_{3}\right\}$

${ }_{427}$ Notice that $Y$ is a non-empty and compact subset of the preimputation set $I^{*}(N \backslash$ $\left.T^{*}, \omega\right)$, and thus, by Schmeidler (1969), the $\operatorname{kernel}^{7}$ of the game $\left(N \backslash T^{*}, \omega\right)$ relative to $Y$ is non-empty, i.e. $\mathcal{K}\left(N \backslash T^{*}, \omega, Y\right) \neq \varnothing$.

\footnotetext{
${ }^{7}$ Notice that the set $Y$ is a non-empty box since it can be rewritten as $Y=\left\{\alpha \in \mathbb{R}^{N \backslash T^{*}}\left|0 \leq \alpha_{i} \leq\right| J^{\theta^{*}} \mid \cdot \varepsilon_{3}\right.$, for all $i \in N \backslash T^{*}$, and $\left.\alpha\left(N \backslash T^{*}\right)=\left|J^{\theta^{*}}\right| \cdot \varepsilon_{3}\right\}$.
} 
Hence, select an element $\delta$ in the kernel of the game $\left(N \backslash T^{*}, \omega\right)$ relative to $Y$, i.e. $\delta \in \mathcal{K}\left(N \backslash T^{*}, \omega, Y\right)$, and define the vector $x \in \mathbb{R}^{N}$ as follows:

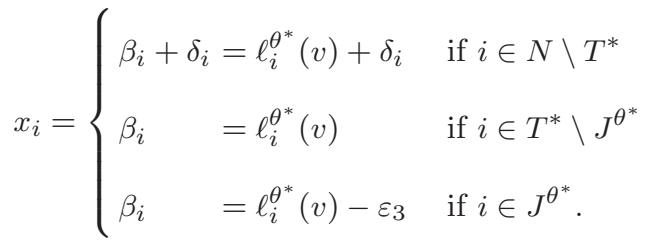

The vector $x$ is an imputation of the game $(N, v)$ : clearly, $x$ is efficient, $x(N)=$ $v(N)$; moreover, by definition of $\varepsilon_{3}$ and (23), we have $x_{i}=\ell_{i}^{\theta^{*}}(v)-\varepsilon_{3} \geq v(\{i\})$, ${ }_{434}$ for all $i \in J^{\theta^{*}}, x_{i}=\ell_{i}^{\theta^{*}}(v) \geq v(\{i\})$, for all $i \in T^{*} \backslash J^{\theta^{*}}$ and, since $\delta_{i} \geq 0$, ${ }_{435} \quad x_{i}=\beta_{i}+\delta_{i}=\ell_{i}^{\theta^{*}}(v)+\delta_{i} \geq v(\{i\})$, for all $i \in N \backslash T^{*}$.

However, it is not in the core of the game $(N, v)$ since $x\left(T^{*} \backslash\left\{i_{t^{*}}\right\}\right)=\ell^{\theta^{*}}(v)\left(T^{*} \backslash\right.$ $\left.\left\{i_{t^{*}}\right\}\right)-\left(\left|J^{\theta^{*}}\right|-1\right) \varepsilon_{3}=v\left(T^{*} \backslash\left\{i_{t^{*}}\right\}\right)-\left(\left|J^{\theta^{*}}\right|-1\right) \varepsilon_{3}<v\left(T^{*} \backslash\left\{i_{t^{*}}\right\}\right)$.

We finally check that $x$ is in the steady bargaining set of the game $(N, v)$. To this aim take $S \subseteq N$ such that $v(S)-x(S)>0$. Notice that, since $x \in I(N, v)$, then

${ }_{440}|S| \geq 2$. Furthermore, it holds that

$$
S \cap J^{\theta^{*}} \neq \varnothing
$$

since otherwise $S \cap J^{\theta^{*}}=\varnothing$ and we would have

$$
\begin{aligned}
v(S)-x(S) & =v(S)-\beta(S)-\delta\left(S \cap\left(N \backslash T^{*}\right)\right) \leq v(S)-\beta(S) \\
& =v(S)-\ell^{\theta^{*}}(v)(S) \leq 0
\end{aligned}
$$

441 reaching a contradiction with $v(S)-x(S)>0$. Next, we shall prove there exists $M \subseteq N$ such that $M \backslash S \neq \varnothing, S \backslash M \neq \varnothing, S \cap M \neq \varnothing$ and $v(M)-x(M) \geq v(S)-x(S)$.

${ }_{443}$ We distinguish two cases.

A: $S \subseteq T^{*}=\left\{i_{1}, \ldots, i_{t^{*}}\right\}$. By the way we have defined $\varepsilon_{3}$, and being $S \subseteq T^{*}$, let us first see that $\ell^{\theta^{*}}(v)(S)=v(S)$. To check it, let us suppose that $\ell^{\theta^{*}}(v)(S)>v(S)$, 
then $v(S)-x(S)=v(S)-\ell^{\theta^{*}}(v)(S)+\left|S \cap J^{\theta^{*}}\right| \cdot \varepsilon_{3} \leq v(S)-\ell^{\theta^{*}}(v)(S)+n \cdot \varepsilon_{3}<0$, which contradicts the hypothesis $v(S)-x(S)>0$. Moreover, by (26), $S \cap J^{\theta^{*}} \neq$ $\varnothing$, and, by Subclaim 1.4, $J^{\theta^{*}} \backslash S \neq \varnothing$. Let $j \in J^{\theta^{*}} \backslash S$ and $i \in J^{\theta^{*}} \cap S$ and take $M=T^{*} \backslash\{i\}$. Notice that $j \in M \backslash S, i \in S \backslash M$ and, since $|S| \geq 2, M \cap S \neq \varnothing$. Furthermore, since $i \in J^{\theta^{*}}$ we have $\ell^{\theta^{*}}(v)(M)=v(M)$, and thus

$$
\begin{aligned}
v(M)-x(M) & =v(M)-\ell^{\theta^{*}}(v)(M)+\left(\left|J^{\theta^{*}}\right|-1\right) \cdot \varepsilon_{3} \\
& =v(S)-\ell^{\theta^{*}}(v)(S)+\left(\left|J^{\theta^{*}}\right|-1\right) \cdot \varepsilon_{3} \geq v(S)-x(S),
\end{aligned}
$$

$444 \quad$ where the inequality follows since $j \in J^{\theta^{*}} \backslash S$.

B: $S \cap\left(N \backslash T^{*}\right) \neq \varnothing$. First let us remark that $S \cap\left(N \backslash T^{*}\right) \neq N \backslash T^{*}$, or equivalently $N \backslash\left(T^{*} \cup S\right) \neq \varnothing$; this holds since, otherwise, $S \cap\left(N \backslash T^{*}\right)=N \backslash T^{*}$ and

$$
\begin{aligned}
v(S)-x(S) & =v(S)-\beta(S)-\delta\left(N \backslash T^{*}\right)=v(S)-\beta(S)-\omega\left(N \backslash T^{*}\right) \\
& =v(S)-\beta(S)-\left|J^{\theta^{*}}\right| \cdot \varepsilon_{3} \\
& =v(S)-\ell^{\theta^{*}}(v)(S)+\left|S \cap J^{\theta^{*}}\right| \cdot \varepsilon_{3}-\left|J^{\theta^{*}}\right| \cdot \varepsilon_{3} \leq 0,
\end{aligned}
$$

reaching a contradiction.

Hence, let $i \in S \cap\left(N \backslash T^{*}\right)$ and select $j \in\left(N \backslash T^{*}\right) \backslash S=N \backslash\left(T^{*} \cup S\right)$ such that

$$
s_{j i}^{\omega}(\delta) \geq s_{i j}^{\omega}(\delta)
$$

Let us prove that such a player $j$ exists. To check it, suppose that, given an arbitrary $k \in\left(N \backslash T^{*}\right) \backslash S$, we would have $s_{k i}^{\omega}(\delta)<s_{i k}^{\omega}(\delta)$. Since $\delta \in \mathcal{K}(N \backslash$ $\left.T^{*}, \omega, Y\right)$ then we would have that either $\delta_{k}=0$ or $\delta_{i}=\left|J^{\theta^{*}}\right| \cdot \varepsilon_{3}$. However, $\delta_{i}=$ $\left|J^{\theta^{*}}\right| \cdot \varepsilon_{3}$ is not possible since, by a similar reasoning as in (27), we would reach a contradiction with $v(S)-x(S)>0$. Therefore, we obtain that $\delta_{k}=0$. Since $k$ was chosen arbitrarily, we would conclude that $\delta_{k}=0$ for all $k \in\left(N \backslash T^{*}\right) \backslash S$ 
and thus

$$
\left|J^{\theta^{*}}\right| \cdot \varepsilon_{3}=\omega\left(N \backslash T^{*}\right)=\delta\left(N \backslash T^{*}\right)=\delta\left(\left(N \backslash T^{*}\right) \cap S\right) .
$$

But then,

$$
\begin{aligned}
v(S)-x(S) & =v(S)-\beta(S)-\delta\left(S \cap\left(N \backslash T^{*}\right)\right) \\
& =v(S)-\ell^{\theta^{*}}(v)(S)+\left|S \cap J^{\theta^{*}}\right| \cdot \varepsilon_{3}-\left|J^{\theta^{*}}\right| \cdot \varepsilon_{3} \leq v(S)-\ell^{\theta^{*}}(v)(S) \leq 0,
\end{aligned}
$$

$449 \quad$ getting a contradiction with $v(S)-x(S)>0$.

Now, by definition and taking agents $i$ and $j$ as in (28), we have

$s_{j i}^{\omega}(\delta)=\omega\left(R^{*}\right)-\delta\left(R^{*}\right)=v\left(R^{*} \cup Q^{*}\right)-\beta\left(R^{*} \cup Q^{*}\right)-\delta\left(R^{*}\right)=v\left(R^{*} \cup Q^{*}\right)-x\left(R^{*} \cup Q^{*}\right)$, for some $R^{*} \subseteq N \backslash T^{*}$, with $j \in R^{*}$ but $i \notin R^{*}$, and some $Q^{*} \subseteq T^{*}$. Hence, by (28), it follows that

$$
\begin{aligned}
v\left(R^{*} \cup Q^{*}\right)-x\left(R^{*} \cup Q^{*}\right) & =s_{j i}^{\omega}(\delta) \geq s_{i j}^{\omega}(\delta) \\
& \geq \omega\left(S \cap\left(N \backslash T^{*}\right)\right)-\delta\left(S \cap\left(N \backslash T^{*}\right)\right) \\
& \geq v(S)-x(S)>0 .
\end{aligned}
$$
Notice that $i \in S \backslash\left(R^{*} \cup Q^{*}\right)$ and $j \in\left(R^{*} \cup Q^{*}\right) \backslash S$. Furthermore, if $S \cap\left(R^{*} \cup Q^{*}\right) \neq$ $\varnothing$, then take $M=R^{*} \cup Q^{*}$ and we are done. Otherwise, in case $S \cap\left(R^{*} \cup Q^{*}\right)=\varnothing$ we have, by $(26),\left(R^{*} \cup Q^{*}\right) \cap J^{\theta^{*}} \neq \varnothing$.

Hence, since we are supposing $S \cap\left(R^{*} \cup Q^{*}\right)=\varnothing,\left(R^{*} \cup Q^{*}\right) \cap J^{\theta^{*}} \neq \varnothing$ and $S \cap J^{\theta^{*}} \neq \varnothing($ see $(26))$, we conclude that

$$
S \cap J^{\theta^{*}} \varsubsetneqq J^{\theta^{*}}
$$

Therefore,

$$
\begin{aligned}
v(S)-x(S) & =v(S)-\beta(S)-\delta\left(S \cap\left(N \backslash T^{*}\right)\right) \\
& =v(S)-\ell^{\theta^{*}}(v)(S)+\left|S \cap J^{\theta^{*}}\right| \cdot \varepsilon_{3}-\delta\left(S \cap\left(N \backslash T^{*}\right)\right) \\
& \leq v(S)-\ell^{\theta^{*}}(v)(S)+\left|S \cap J^{\theta^{*}}\right| \cdot \varepsilon_{3} \\
& \leq\left(\left|J^{\theta^{*}}\right|-1\right) \cdot \varepsilon_{3} .
\end{aligned}
$$


Hence, it easily follows that

$$
v(S)-x(S) \leq\left(\left|J^{\theta^{*}}\right|-1\right) \cdot \varepsilon_{3}=v\left(T^{*} \backslash\{k\}\right)-x\left(T^{*} \backslash\{k\}\right)
$$
getting a contradiction with the hypothesis $C(N, v)=\mathcal{S B}(N, v)$. 